\title{
E-Fields of Electrode-Based Through-The-Earth (TTE) Communication
}

\author{
Lincan Yan*, Chenming Zhou, Reyes Miguel, Bruce Whisner, Nicholas Damiano \\ Pittsburgh Mining Research Division (PMRD) \\ National Institute for Occupational Safety and Health (NIOSH) \\ 626 Cochrans Mills Road \\ Pittsburgh, PA 15236, USA
}

\begin{abstract}
An electrode-based through-the-earth (TTE), or linear TTE, communication system sends its signal directly through the earth overburden of a mine by driving an $\mathrm{AC}$ current into the earth. The resultant current, present at the receiver, is detected as a voltage and communication is established. The electrode-based TTE system may achieve a considerably large transmission range $>305 \mathrm{~m}(1000 \mathrm{ft})$. This kind of system may be implemented for emergency communication close to an area such as a refuge alternative or other strategic location to establish communication between underground miners and the surface. Since it does not rely on extensive infrastructure underground, this communication would provide an alternate path out of the mine. Given the attenuation characteristics of the earth-which is the major factor determining the performance of TTE systems-the receiver sensitivity, transmitter antenna length and orientation, working frequency, and transmitted current required for communication can be estimated and determined. In an effort to estimate the earth attenuation, the analytic solution for the electrical field distribution of an electrode-based TTE communication system in a homogenous half-space is derived. A prototype system was built and tested at a mine site. The test data is compared with the modeling results, and a close agreement is found between them.
\end{abstract}

Keywords -- Through-the-earth (TTE); electrode-based TTE; extremely low frequency (ELF); mine communication.

\section{INTRODUCTION}

One of the requirements of the Mine Improvement and New Emergency Response Act (MINER Act) of 2006 is the installation of post-accident, two-way communications and electronic tracking systems for all underground coal mines. As one of the through-the-earth (TTE) wireless communication methods available for the mining industry, the electrode-based, or linear, TTE systems establish communication by injecting electric current through the overburden at the transmitter antenna (TX) and detecting the induced voltage at the receiver antenna (RX). During the communication, signals are either sent from underground to surface (uplink) or from surface to underground (downlink). In order to achieve a successful communication link, it is necessary to understand the electric fields within the earth used for the downlink transmission so that the appropriate size transmitting antenna, TX/RX relative orientation, and operating frequency can be chosen. In prior research, the E-fields of electric line transmitter were obtained for a static TX current [1] [2]. A 2-D model was developed for the subsurface electromagnetic fields of an infinite currentcarrying cable on the surface of a homogeneous earth [3]. In any real mine communication, however, the cable (TX) is of finite length.

In this paper, the underground electric fields of electrodebased TTE downlink communication with finite cable length were developed based on Hill and Wait's work [4]. Using its prototype electrode-based TTE system, the National Institute for Occupational Safety and Health (NIOSH) conducted experiments at a coal mine. The objectives were to evaluate the path loss and optimize the working frequency that has the smallest attenuation at a specific mine. Furthermore, the theory solution was compared to the experimental data.

\section{E-FIELDS FOR DOWNLINK COMMUNICATION}

A current-carrying cable in the presence of a homogenous half-space is depicted as in Fig. 1. The cable is $x$-directed and with length of $2 l$ with source current running between $-l$ and $l$ on the $x$ axis. The current has a magnitude of $I$ and has the form of $e^{j \omega t}$, where $\omega$ is the angular frequency and $j$ is the square root of -1 . All displacement currents are negligible in that $\omega$ is in the very low or extra low frequency range. The Hertz vector due to an infinitesimal length source $d x^{\prime}$ located at $x^{\prime}$ is given by [5]:

$$
\begin{gathered}
d \Pi_{x}=\frac{I d x^{\prime}}{2 \pi \sigma \gamma^{2}}\left(\frac{\partial^{2} P}{\partial z^{2}}-\frac{\partial^{3} N}{\partial z^{3}}+\gamma^{2} \frac{\partial N}{\partial z}\right) \\
d \Pi_{y}=0 \\
d \Pi_{z}=\frac{I d x^{\prime}}{2 \pi \sigma \gamma^{2}}\left(\frac{\partial^{3} N}{\partial x \partial z^{2}}-\frac{\partial^{2} P}{\partial x \partial z}\right)
\end{gathered}
$$

where $N=I_{0}[(R+z) \gamma / 2] K_{0}[(R-z) \gamma / 2], \quad P=e^{-\gamma R} / R$, $\gamma^{2}=j \omega \mu_{0} \sigma, \rho=\sqrt{y^{2}+\left(x-x^{\prime}\right)^{2}}, R=\sqrt{\rho^{2}+z^{2}}, \mu_{0}$ is the permeability of free space, $\sigma$ is the earth conductivity, $I_{0}$ is the modified Bessel function of the first kind of order zero with the argument of $(R+z) \gamma / 2$, and $K_{0}$ is the modified Bessel function of the second kind of order zero with the argument of $(R-z) \gamma / 2$.

\footnotetext{
* Corresponding author. Email address: LYan1@cdc.gov.
} 
The electric field components are derived through

$$
\boldsymbol{E}=-\gamma^{2} \boldsymbol{\Pi}+\nabla(\nabla \cdot \boldsymbol{\Pi})
$$

After some simplification, the three components in a Cartesian coordinates system can be expressed as

$$
\begin{gathered}
d E_{x}=\frac{-I d x^{\prime}}{2 \pi \sigma}\left(\frac{\partial^{2} P}{\partial z^{2}}+\frac{\partial^{3} N}{\partial y^{2} \partial z}\right) \\
d E_{y}=\frac{I d x^{\prime}}{2 \pi \sigma}\left(\frac{\partial^{3} N}{\partial x \partial y \partial z}\right) \\
d E_{z}=\frac{-I d x^{\prime}}{2 \pi \sigma}\left(\frac{\partial^{2} P}{\partial x \partial z}\right)
\end{gathered}
$$

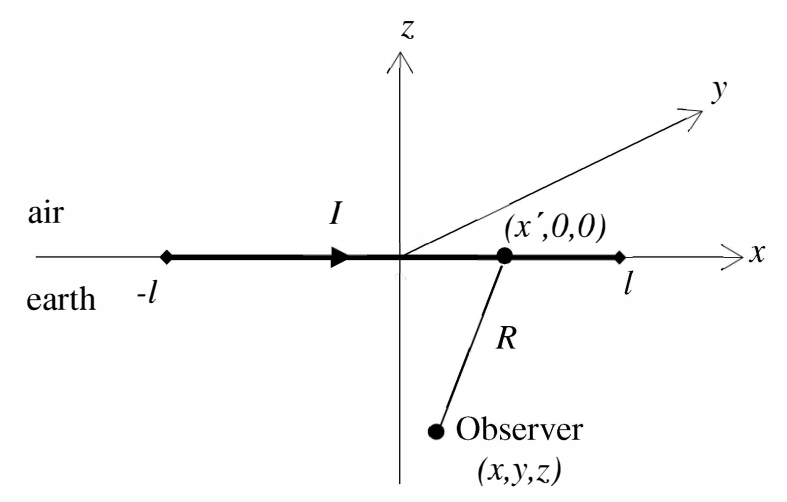

Fig. 1. Finite length cable in the presence of a homogeneous half-space.

The expression for the partial derivatives of $P$ and $N$ are listed as below, with some corrections to what were given in [4].

$$
\begin{gathered}
\frac{\partial^{2} P}{\partial z^{2}}=\frac{e^{-\gamma R}}{R^{2}}\left(\frac{3 z^{2}}{R^{3}}+\frac{3 \gamma z^{2}}{R^{2}}-\frac{1}{R}+\frac{\gamma^{2} z^{2}}{R}-\gamma\right) \\
\frac{\partial^{3} N}{\partial x \partial y \partial z}=A_{00} I_{0} K_{0}+A_{11} I_{1} K_{1}+A_{01} I_{0} K_{1} \\
\quad+A_{10} I_{1} K_{0} \\
\frac{\partial^{3} N}{\partial y^{2} \partial z}=\frac{\gamma}{2}\left(B_{00} I_{0} K_{0}+B_{11} I_{1} K_{1}+B_{01} I_{0} K_{1}\right. \\
\left.\quad+B_{10} I_{1} K_{0}\right) \\
\frac{\partial^{2} P}{\partial x \partial z}=\frac{e^{-\gamma R}\left(x-\mathrm{x}^{\prime}\right) z}{R^{3}}\left(\frac{3}{R^{2}}+\frac{3 \gamma}{R}+\gamma^{2}\right)
\end{gathered}
$$

where

$$
\begin{aligned}
& A_{00}=\frac{-3\left(x-x^{\prime}\right) y z \gamma^{2}}{2 R^{4}} \\
& A_{11}=\frac{y z \gamma^{2}\left(x-x^{\prime}\right)\left(5 R^{2}-3 z^{2}\right)}{2 R^{4}\left(R^{2}-z^{2}\right)} \\
& A_{01}=-\frac{y \gamma\left(x-x^{\prime}\right)\left(3(z-R)+z R^{2} \gamma^{2}\right)}{2 R^{5}} \\
& A_{10}=\frac{y \gamma\left(x-x^{\prime}\right)\left(3(z+R)+z R^{2} \gamma^{2}\right)}{2 R^{5}} \\
& B_{00}=\frac{\gamma z}{R^{2}}\left(1-\frac{3 y^{2}}{R^{2}}\right)
\end{aligned}
$$

$$
\begin{aligned}
& B_{11}=\frac{\gamma z}{R^{2}}\left(-1+\frac{3 y^{2}}{R^{2}}+\frac{2 y^{2}}{\rho^{2}}\right) \\
& B_{01}=\frac{1}{R^{2}}\left(-1+\frac{z\left(1-\gamma^{2} y^{2}\right)}{R}+\frac{3 y^{2}}{R^{2}}-\frac{3 z y^{2}}{R^{3}}\right) \\
& B_{10}=\frac{1}{R^{2}}\left(-1-\frac{z\left(1-\gamma^{2} y^{2}\right)}{R}+\frac{3 y^{2}}{R^{2}}+\frac{3 z y^{2}}{R^{3}}\right)
\end{aligned}
$$

and $I_{1}$ is the modified Bessel functions of the first kind of order one, with the argument of $(R+z) \gamma / 2 ; K_{1}$ is the modified Bessel functions of the second kind of order one, with the argument of $(R-z) \gamma / 2$.

The electric fields at observation point $(x, y, z)$ then can be obtained by integrating (5) through (7) over the range of $x^{\prime}$ from $-l$ to $l$. The electric fields-hence the transmitted signal strength picked up by the RX - are affected by several factors as described below.

\section{Conductivity}

The E-fields were normalized by the magnitude of the transmitting current to eliminate the effect of transmitter current on receiving voltage at RX. The normalized horizontal E-field at an observation point located at $100 \mathrm{~m}(328 \mathrm{ft})$ directly below the midpoint of the TX as a function of conductivity for various operating frequencies is given in Fig. 2. As shown in the plot, the E-field strength decreases monotonically with increasing conductivity for all frequencies in the range. The decreasing rate, however, is lower at lower frequency than at higher frequency. It becomes obvious that low operating frequency should be chosen for mines with high conductivity in order to achieve the E-field strength required for a successful communication link.

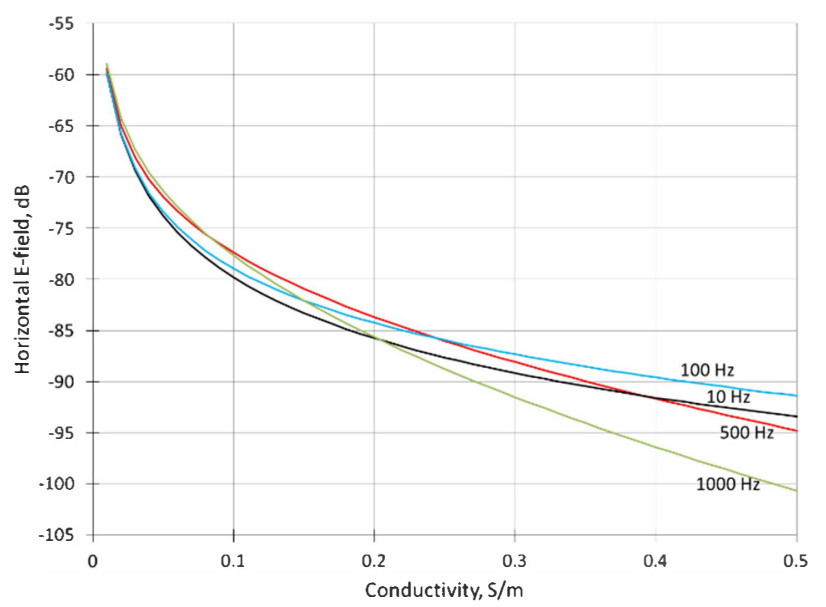

Fig. 2. Normalized horizontal E-field changes with conductivity for various frequencies. $x=0, y=0, z=-h=-100 \mathrm{~m}$.

\section{Overburden Depth}

As shown in Fig. 3, the overburden depth has a significant effect on received E-field strength. Signal strength drops rapidly at deep mines. However, an electrode-based TTE 
communication link can still be established if the operating frequency is chosen properly for deep mines.

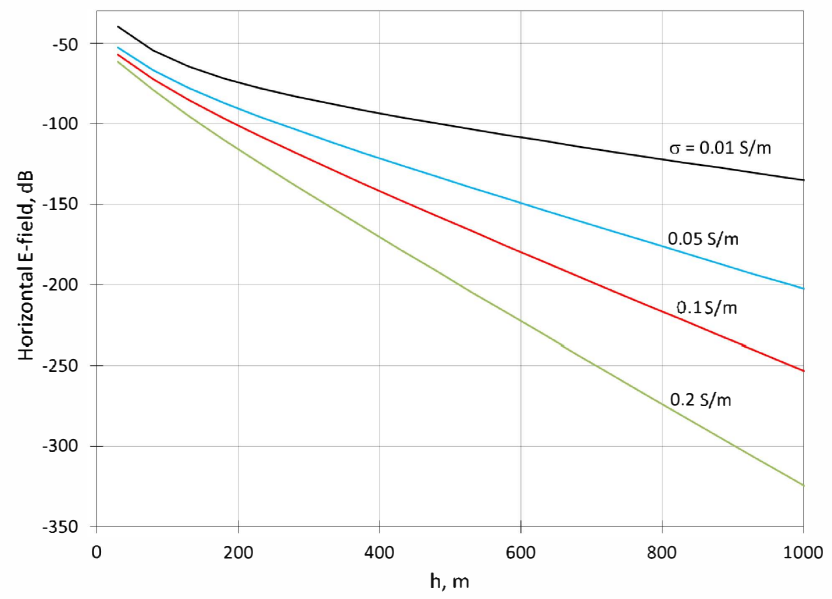

(a)

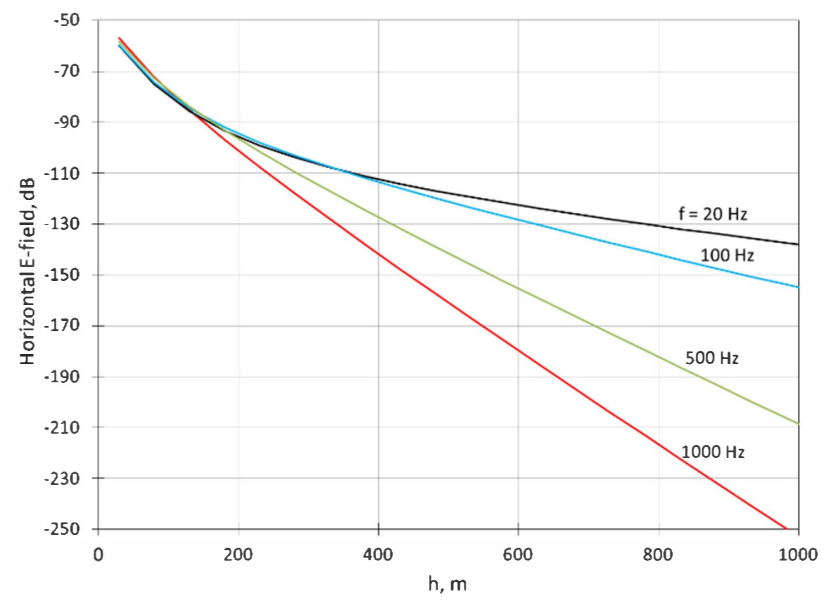

(b)

Fig. 3. Normalized horizontal E-field changes with overburden depth $(h)$ for a) various conductivities at $1 \mathrm{kHz}$ and $\mathrm{b}$ ) various frequencies at $0.1 \mathrm{~S} / \mathrm{m} . x=0$, $y=0$.

\section{TX/RX Antenna Length}

The transceiver in electrode-based TTE communication systems usually has one wire antenna (grounded cable) that is used for both TX and RX. As reflected in Fig. 4, increasing the antenna length will enhance the E-field at the receiver, given all other parameters are unchanged. But that enhancement will stop when the $l / h$ value is getting larger ( $>0.8)$.

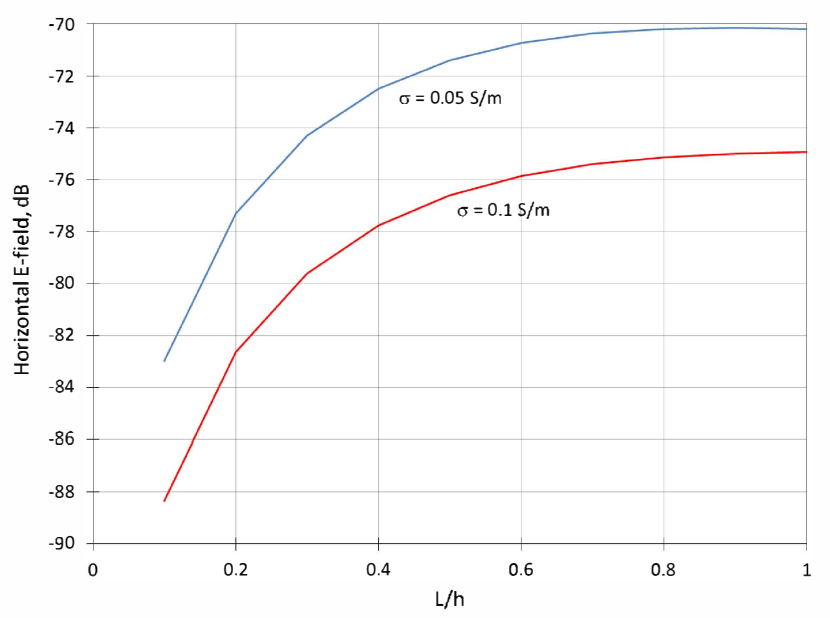

Fig. 4. Normalized horizontal E-field changes with TX/RX length ( $2 l$ ). $x=0$, $y=0, z=-h=-100 \mathrm{~m}, f=300 \mathrm{~Hz}$.

\section{Frequency}

The normalized horizontal E-field at an observation point located at $100 \mathrm{~m}(328 \mathrm{ft})$ directly below the midpoint of the TX is plotted with operating frequency for various earth conductivities in Fig. 5. All E-field components vanish except $x$-component at this point. According to Fig. 5, frequency will have a different effect on E-field strength. Increasing frequency will increase induced voltage at RX for low conductivity $(<0.01 \mathrm{~S} / \mathrm{m})$. However, there is an "optimal" frequency existing if the conductivity has a higher value (> $0.05 \mathrm{~S} / \mathrm{m})$. That "optimal" frequency shifts towards lower frequencies as the conductivity increases. That "optimal" frequency or peak is more pronounced for higher conductivities.

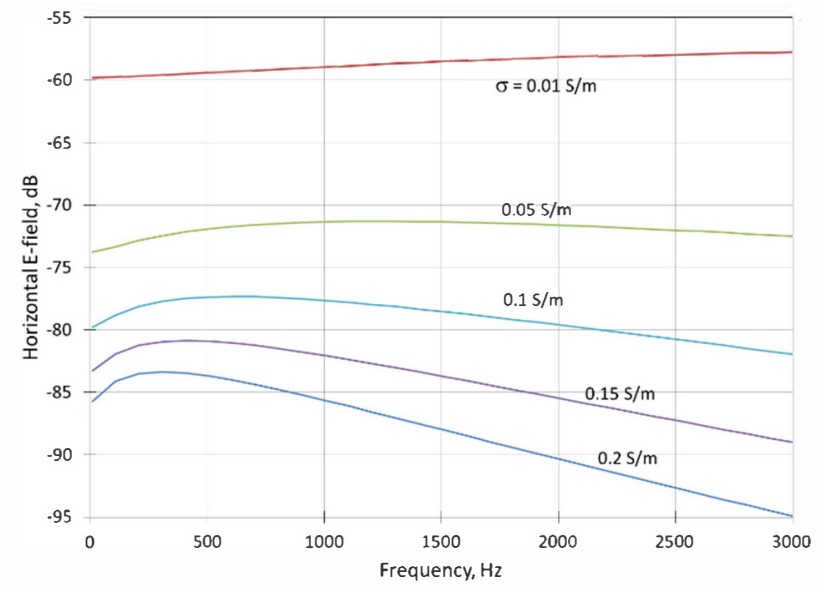

Fig. 5. Underground normalized horizontal E-field changes with operating frequencies for various earth conductivities. $x=0, y=0, z=-h=-100 \mathrm{~m}$.

For extremely low frequency (ELF) range $(<30 \mathrm{~Hz})$ or DC excitation source, $\gamma=j \omega \mu_{0} \sigma \rightarrow 0$. The Hertz components in [5] then can be simplified as

$$
d \Pi_{x}=\frac{I d x^{\prime}}{4 \pi \sigma} \frac{1}{R}
$$




$$
\begin{gathered}
d \Pi_{y}=0 \\
d \Pi_{z}=\frac{-I d x^{\prime}}{4 \pi \sigma} \frac{\left(x-x^{\prime}\right)}{\rho^{2}}(1+z / R)
\end{gathered}
$$

The E-fields due to an infinitesimal length source $d x^{\prime}$ located at $x^{\prime}$ are given by taking the operation in (4) and setting $\gamma=0$, i.e.,

$$
\begin{aligned}
& d E_{x}=\frac{I\left(2 x^{2}-4 x x^{\prime}+2 x^{\prime 2}-y^{2}-z^{2}\right)}{2 \pi \sigma\left(x^{2}-2 x x^{\prime}+x^{\prime 2}+y^{2}+z^{2}\right)^{5 / 2}} d x^{\prime} \\
& d E_{y}=\frac{3 I\left(x-x^{\prime}\right) y}{2 \pi \sigma\left(x^{2}-2 x x^{\prime}+x^{\prime 2}+y^{2}+z^{2}\right)^{5 / 2}} d x^{\prime} \\
& d E_{z}=\frac{3 I\left(x-x^{\prime}\right) z}{2 \pi \sigma\left(x^{2}-2 x x^{\prime}+x^{\prime 2}+y^{2}+z^{2}\right)^{5 / 2}} d x^{\prime}
\end{aligned}
$$

So, for ELF or DC excitation source, the E-fields at the underground observer point $(x, y, z)$ are obtained by integrating (15) - (17) over the entire TX length from $-l$ to $l$.

$$
\begin{aligned}
& E_{x} \\
& =\frac{I}{2 \pi \sigma}\left(\frac{-l+x}{\left(l^{2}-2 l x+x^{2}+y^{2}+z^{2}\right)^{3 / 2}}\right. \\
& \left.-\frac{l+x}{\left(l^{2}+2 l x+x^{2}+y^{2}+z^{2}\right)^{3 / 2}}\right) \\
& E_{y} \\
& =\frac{I y}{2 \pi \sigma}\left(\frac{1}{\left(l^{2}-2 l x+x^{2}+y^{2}+z^{2}\right)^{3 / 2}}\right. \\
& \left.-\frac{1}{\left(l^{2}+2 l x+x^{2}+y^{2}+z^{2}\right)^{3 / 2}}\right) \\
& E_{z} \\
& =\frac{I z}{2 \pi \sigma}\left(\frac{1}{\left(l^{2}-2 l x+x^{2}+y^{2}+z^{2}\right)^{3 / 2}}\right. \\
& \left.-\frac{1}{\left(l^{2}+2 l x+x^{2}+y^{2}+z^{2}\right)^{3 / 2}}\right)
\end{aligned}
$$

Note that (18) and (19) are exactly the same as what were given in [1] and [2].

\section{EXPER IMENT AT MINE}

\section{Test Setup}

NIOSH conducted experiments at a coal mine to test a prototype electrode-based TTE system (Fig. 6). The prototype system, a transceiver (TCVR), consists of a signal generator, control software and modules, power amplifier, a pair of electrodes serving as both TX and RX antenna, 12-volt batteries, and a data acquisition computer. The TX current through the wire antenna was calculated by measuring and converting the voltage output of a passive current probe (model no. BCP-510 by AH Systems). The output of the receive antenna was buffered and amplified by a preamplifier. The signal was then digitized by a National Instruments NI9239 analog input module at 50,000 samples per second and processed by LabView software. The LabView software computed a 50,000 point Hanning window Fast Fourier Transform (FFT) voltage spectrum for each channel, yielding a $1 \mathrm{~Hz}$ bin resolution. The FFT datum of the frequency under test was displayed on a real-time waterfall diagram for visual interpretation, while the entire time domain data series were stored to a hard disk for post-processing.

Two identical systems were built so that signals could be transmitted and received at both surface and underground. Both system circuits were fused for safety concerns.

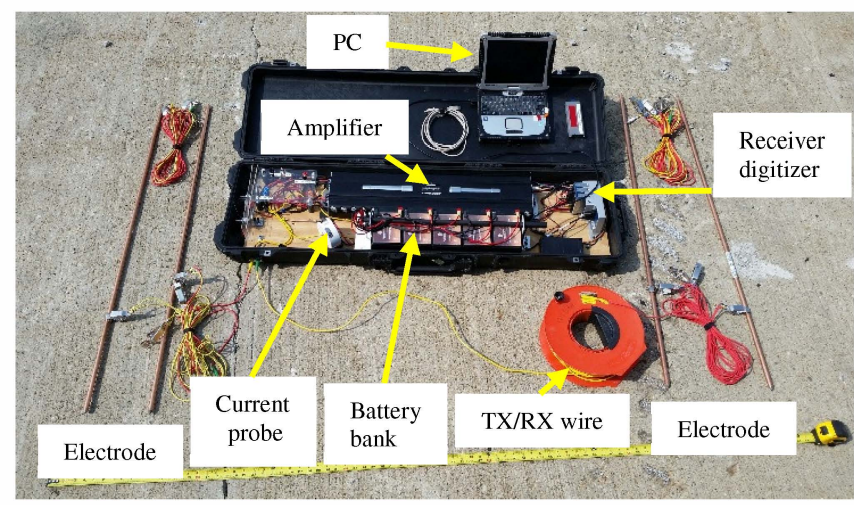

Fig. 6. NIOSH developed electrode-based TTE prototype system.

Two surface locations (AG \#1 and AG \#2) and two underground locations (UG \#1 and UG \#2) were selected to set up the electrodes and system. The above-ground test locations $A G \# 1$ and $A G \# 2$ were chosen to be directly above UG \#1 and UG \#2, respectively. AG \#1 was $149 \mathrm{~m}$ (490 ft) directly above UG \#1, and AG \#2 was $293 \mathrm{~m}$ (961ft) directly above UG \#2. The horizontal separation distance between UG \#1 and UG \#2 (or between AG \#1 and AG \#2) was about $2134 \mathrm{~m}(7,000 \mathrm{ft}$ ). Those test locations are depicted in Fig. 7. For all tests, the surface TX wire antenna is parallel to the underground RX wire antenna and vice versa.

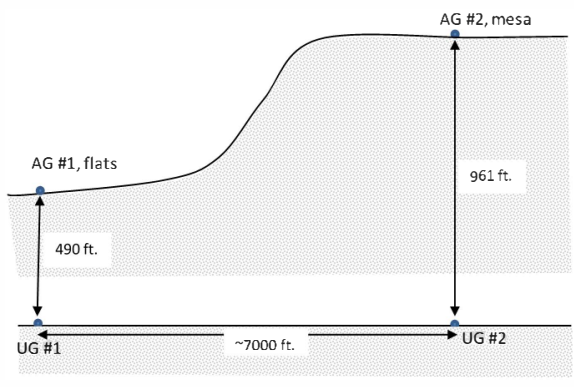

Fig. 7. Underground and surface test locations.

Due to space constraints, two different grounding rod configurations were used for underground and surface test locations (Fig. 8 and Fig. 9). Eight (8) 1.59-cm (5/8-inch) holes up to $1.22 \mathrm{~m}(4 \mathrm{ft})$ deep were drilled at each electrode site. A solution of copper (II) sulfate $\left(\mathrm{CuSO}_{4}\right)$ was added into all the holes to improve the conductivity with earth. A copper rod was then inserted into each of the drilled holes. Based on our previous research, it was expected that 8 rods would be sufficient for the transmitter (TX) to achieve a low resistance connection to the earth. The two electrodes were separated by

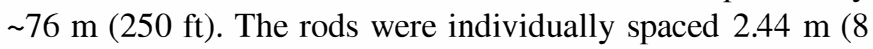


$\mathrm{ft}$ ) in both configurations. The system was connected to the electrodes. That pair of electrodes acted as both a transmit antenna during transmission, and as a receive antenna during receiving. For all the tests, underground and surface antenna wires were located in the $x z$ plane and aligned with the $x$-axis (mine entry direction) and parallel to each other.

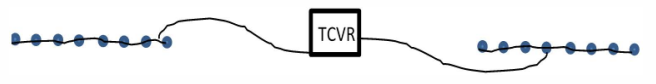

(a)

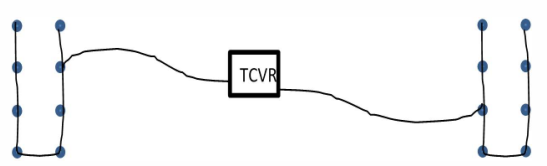

(b)

Fig. 8. Grounding rods (blue dots) configuration with transceiver (TCVR) deployed at (a) underground and (b) surface.

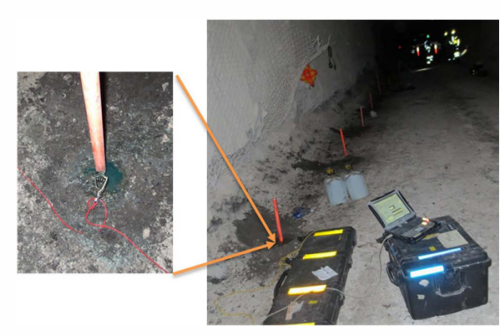

(a)

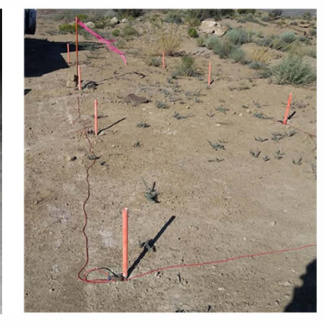

(b)
Fig. 9. Grounding rod connection at (a) underground and (b) surface.

\section{Test Results}

Five frequencies were used during the tests: 20, 100, 330, 990 , and $3030 \mathrm{~Hz}$. Two frequencies $(450 \mathrm{~Hz}$ and $610 \mathrm{~Hz})$ were added for some tests to look at the optimal frequency in range of 100-1000 Hz. Four tests were conducted in successionvertically downlink/uplink and diagonally downlink/uplink. The E-field strength observed by RX will depend on several factors such as magnitude of TX current, length of TX and RX, separation distance of TX and RX, relative orientation of TX and RX, and earth electrical properties [2]. With some of the factors as discussed in Section II, only the TX current magnitude, operating frequency, and overburden depth were changed during the tests.

The ground resistance between two electrodes (two groups of copper rods) was measured at the four test locations before the test to determine the voltage that would be applied to the TX. As shown in Table 1, those locations have relatively low resistance, especially at AG\#1 and UG \#1. For an electrodebased TTE communication system installed in a specific mine, that low ground resistance is favored by the system since a greater transmit current, and hence greater E-field strength, can be achieved for a given TX voltage. Note that the ground resistance was measured between the pair of TX electrodes, while the earth conductivity was an estimate value for the earth medium between TX and RX.

\begin{tabular}{ccccc}
\multicolumn{5}{c}{ Table 1. Ground resistance at test locations. } \\
\hline \hline location & AG \#1 & AG \#2 & UG \#1 & UG \#2 \\
\hline $\begin{array}{c}\text { ground resistance } \\
\text { (Ohms) }\end{array}$ & 1.8 & 35 & 2.0 & 7.0
\end{tabular}

Four transmission tests between the surface and underground were conducted. The test results are shown and described as below.

\subsection{Vertical Transmission between $U G \# 1$ and AG \#1}

The underground unit was located at location UG \#1, while the surface unit was located at location AG \#1. The surface unit transmitted at various TX current magnitudes (labelled as V1 through V4 in descending order in Fig. 10) for five frequencies of 20,100,330,990, and $3030 \mathrm{~Hz}$. As shown in Fig. 10, the signal reception is very repeatable for various TX currents. The underground unit received the signal for all five frequencies (Fig. 10a). Given the same TX current magnitude, the received signal peaked in the $100-500 \mathrm{~Hz}$ frequency range. The uplink transmission, however, was not successful for 3030 Hz.

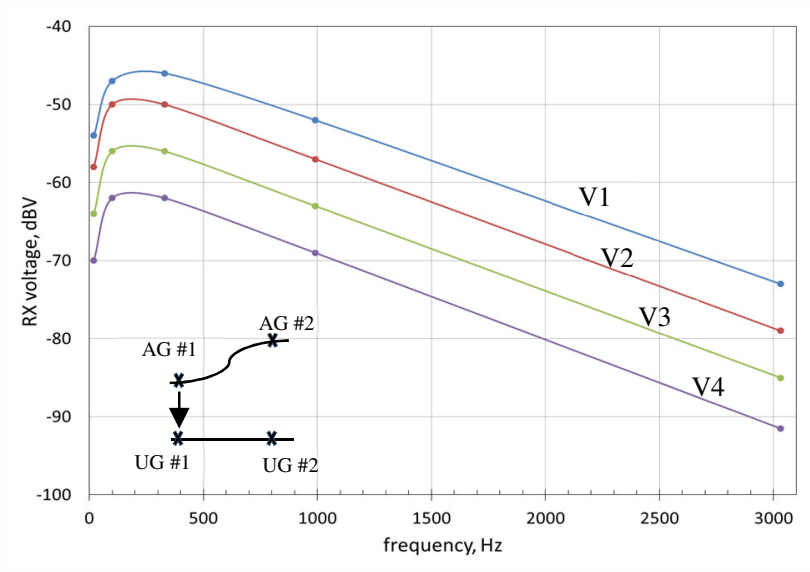

(a)

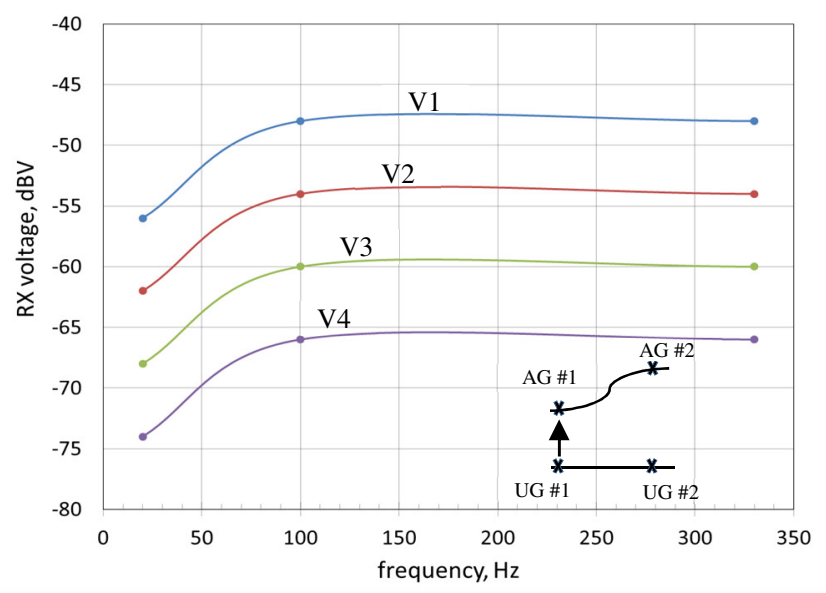

(b) 
Fig. 10. Signal strength at RX changing with operating frequency for a) downlink and b) uplink at locations AG \#1 and UG \#1 at various TX amplifier voltage outputs (V1 through V4).

\subsection{Diagonal Transmission between $U G \# 1$ and AG \#2}

The underground unit was kept at location UG\#1, while the surface unit was moved to location AG \#2. The surface unit transmitted signals at various TX current magnitudes for five frequencies of 20, 100, 330, 990, and $3030 \mathrm{~Hz}$. However, only two frequencies, 20 and $100 \mathrm{~Hz}$, could be received for the uplink transmission at those two locations. No signal was detected for the downlink transmission. This was due to the higher ground resistance at AG \#2 (mesa area) and hence the low current that the transmitter can achieve at maximum input voltage.

\subsection{Vertical Transmission between $U G$ \#2 and AG \#2}

The underground unit was then moved to location UG \#2, while the surface unit was at location AG \#2. The surface unit transmitted at various TX current magnitudes for seven frequencies of 20,100, 330, 450, 610, 990, and $3030 \mathrm{~Hz}$. The underground unit received signals for the first five low frequencies of 20,100,330, 450, and $610 \mathrm{~Hz}$ (Fig. 11). Given the same TX current magnitude, the received signal peaks at approximately $100 \mathrm{~Hz}$. Similarly, in the downlink transmission, the signal for the uplink also peaks at approximately $100 \mathrm{~Hz}$. The signal for the uplink is about 9 $\mathrm{dBV}$ greater than that for the downlink given the same transmitting voltage. The explanation is that the resistance at location $A G \# 2$ is much higher than that at location UG \#2, so the resultant TX current is much lower at AG \#2 than that at UG \#2.

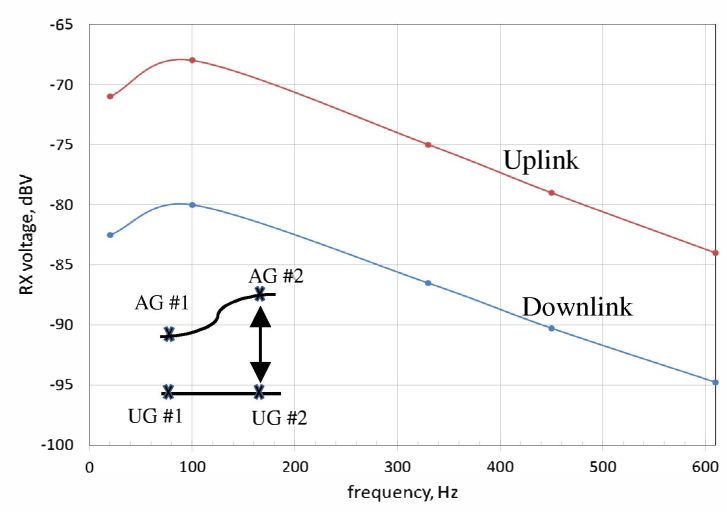

Fig. 11. Signal strength at RX changing with operating frequency for downlink and uplink at locations AG \#2 and UG \#2.

\subsection{Diagonal Transmission between UG \#2 and AG \#1}

The underground unit was kept at location UG \#2, while the surface unit was moved to location $A G \# 1$. The surface unit transmitted at various TX current magnitudes for seven frequencies of 20, 100, 330, 450, 610, 990, and $3030 \mathrm{~Hz}$. The surface unit was able to receive signals for the first three low frequencies of 20, 100, and $330 \mathrm{~Hz}$ (Fig. 12). Given the same
TX current magnitude, the received signal peaks at approximately $100 \mathrm{~Hz}$. For the downlink transmission, the underground unit received signal for the first four low frequencies of $20,100,330$, and $450 \mathrm{~Hz}$. The higher frequencies seemed to be unable to penetrate the earth, probably due to the signal attenuation in both directions.

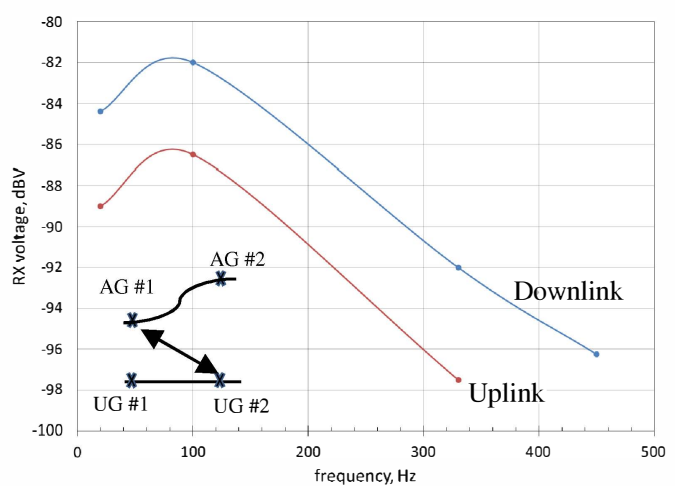

Fig. 12. Signal strength at RX changing with operating frequency for a) uplink and b) downlink between $U G \# 2$ and $A G \# 1$.

\subsection{Signal Reciprocity}

For magnetic loop TTE communications, the electromagnetic propagation is non-reciprocal [6] [7], especially when the apparent earth conductivity has a high value. Based on a homogenous half-space model, Hill [8] and Hill and Wait [4] gave the same field distribution for the uplink transmission as that for the downlink transmission for the electrode-based TTE communication, given the same transmitter configuration. Table 2 shows the normalized measured $\mathrm{RX}$ voltage $\left(V_{\mathrm{RX}} / I_{\mathrm{TX}}\right)$ at all test locations for all available frequencies. The difference between uplink and downlink transmission is less than $1 \%$ for all cases. The test data confirms that the transmission is reciprocal for electrodebased TTE communication at that mine site.

Table 2. Normalized RX voltage for uplink and downlink transmission at various test locations.

\begin{tabular}{c|c|c|c|c}
\hline \hline $\begin{array}{c}\text { link } \\
\text { between }\end{array}$ & $\begin{array}{c}\text { Frequency, } \\
\mathrm{Hz}\end{array}$ & $\begin{array}{c}V_{\mathrm{RX}} / I_{\mathrm{TX}}, \mathrm{dBV} \\
\text { downlink }\end{array}$ & $\begin{array}{c}V_{\mathrm{RX}} / I_{\mathrm{TX}}, \mathrm{dBV} \\
\text { uplink }\end{array}$ & difference, \% \\
\hline \multirow{3}{*}{$\begin{array}{c}\text { AG\#1 and } \\
\text { UG\#1 }\end{array}$} & 20 & -81.4 & -81.3 & 0.1 \\
\cline { 2 - 5 } & 100 & -74.6 & -74.5 & 0.2 \\
\cline { 2 - 5 } & 330 & -74.3 & -74.4 & 0.1 \\
\cline { 2 - 5 } & 20 & -91.6 & -91.9 & 0.3 \\
\cline { 2 - 5 } & 100 & -90.5 & -90.0 & 0.5 \\
\cline { 2 - 5 } AG\#2 and & 330 & -97.1 & -96.9 & 0.2 \\
\cline { 2 - 5 } & 450 & -100.2 & -100.2 & 0.0 \\
\hline \multirow{3}{*}{$\begin{array}{c}\text { AG\#1 and } \\
\text { UG\#2 }\end{array}$} & 610 & -105.2 & -105.6 & 0.4 \\
\cline { 2 - 5 } & 20 & -111.4 & -112.4 & 0.8 \\
\cline { 2 - 5 } & 100 & -110.1 & -110.9 & 0.7 \\
\hline
\end{tabular}

\section{MODEl VAlidation}

The E-field strength observed by the RX is a critical factor which determines whether an electrode-based TTE communication link can be established or fails. Predicting the E-fields at a mine with specific parameters such as overburden depth and conductivity helps to evaluate the feasibility of 
installation of such a system at that mine, choose proper installation locations, estimate the required TX antenna length and current/voltage, and use the proper TX/RX orientation.

The test data for vertical downlink transmission at locations AG \#1 and UG \#1 was compared with the analytical solution (Fig. 13). The RX voltage $\left(V_{R X}\right)$ was normalized by TX current $\left(I_{T X}\right)$ to eliminate the source effect, and averaged over all tests with different TX output voltage. For the analytical solution, the RX voltage was obtained by numerically integrating (5) over the entire length of the RX wire antenna. Both test data and model calculation show peaks at the frequency range of 20-500 Hz. This suggests that an electrode-based TTE system operating at this frequency range may gain the strongest signal at this mine site.

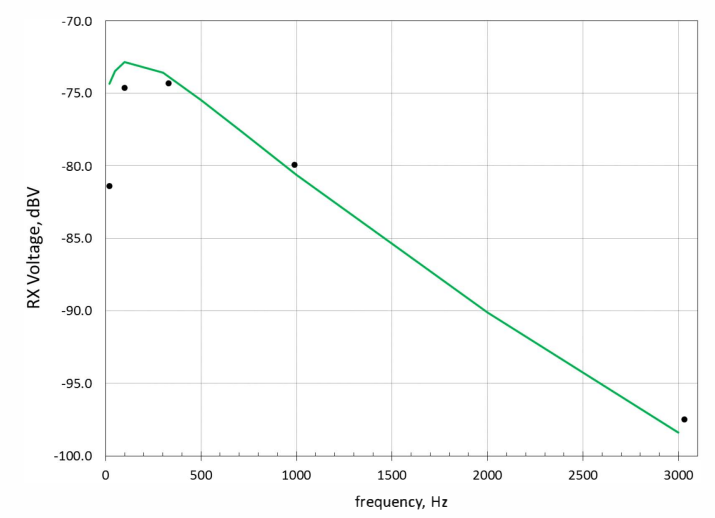

Fig. 13. Normalized RX voltage $\left(\boldsymbol{V}_{\boldsymbol{R} X} / \boldsymbol{I}_{T X}\right)$ for test data (dots) and theoretical prediction (solid line). $\sigma=0.2 \mathrm{~S} / \mathrm{m}, h=-149 \mathrm{~m}, l=38 \mathrm{~m}$.

\section{CONCLUSION}

The analytic solution for the electric field distribution within the earth medium for electrode-based TTE communication was developed. Based on those solutions, several factors such as conductivity, length of TX/RX antenna, overburden depth, and operating frequency were discussed for their effects on E-field strength at the observer point. The explicit forms of E-field components were derived for the ELF or DC excitation case. The analytic result was then validated with test data. A close agreement can be found between them.

To obtain the E-field strength required for establishing a communication link, the TX current must be increased by either increasing the amplifier output voltage or decreasing the ground resistance between the pair of electrodes of the TX. While increasing the amplifier output voltage is generally limited by safety issues, the ground conductivity between the electrodes can be improved by adding a conductive solution to the earth surrounding the electrodes (grounding rods). According to our research, the ground conductivity can be increased up to $50 \%$ by adding a copper (II) sulfate solution to the electrodes.

As mentioned earlier, increasing the TX/RX antenna length will enhance the E-field before reaching its limit. The TX/RX relative orientation, on the other hand, will also change the E- field distribution pattern in the earth medium. More details on the effect of TX/RX relative orientation on E-field distribution can be found in [2]. In this paper, we only consider RX parallel to TX.

The NIOSH electrode-based, prototype TTE system was tested and transmission data was acquired at a coal mine. This data is an important part of the understanding of this kind of TTE system performance. An interesting finding is that the ground resistance at some tested locations at this mine is surprisingly low (as low as a few ohms for up to $76 \mathrm{~m}$ or 250 $\mathrm{ft}$ separation distance). Low resistance is favorable for an electrode-based TTE system because less voltage is needed to create the desired current. The test data also confirms that the transmission between surface and underground is reciprocal for electrode-based TTE communication given the relatively large separation of the TX and RX antennas in our tests. During the test, text messages were transmitted rather than continuous waveform transmission. As a result of the low resistance, we successfully communicated text messages from underground to the surface with a horizontal separation distance of $\sim 2134 \mathrm{~m}(7000 \mathrm{ft})$. The performance of the prototype electrode-based TTE system was very repeatable at these locations.

Finally, the validated model can be used to optimize the operating frequency, to estimate the required transmitting current, and to predict the performance of an electrode-based TTE system at a mine based on its geologic characteristics such as earth conductivity, overburden depth, etc.

\section{ACKNOWLEDGEMENT}

The authors would like to thank Dr. J. A. Waynert for his useful comments.

\section{DISCLAIMER}

Mention of a company name or product does not constitute an endorsement by the National Institute for Occupational Safety and Health. The findings and conclusions in this report are those of the authors and do not necessarily represent the views of the National Institute for Occupational Safety and Health. This article is not subject to US copyright law.

\section{REFERENCES}

[1] R. Geyer, G. Keller and T. Ohya, "Research on the transmission of electromagnetic signals between mine workings and the surface," Colorado School of Mines, 1974.

[2] L. Yan and C. Sunderman, "Electric field of grounded horizontal line transmitter for through the earth communication," in the 31st International Review of Progress in Applied Computational Electromagnetics, Willamsburg, WV, 2015. 
[3] J. Wait and K. Spies, "Subsurface electromagnetic fields of a line source on a conducting half-space," Radio Science, vol. 6, no. 8-9, p. 781-786, 1971.

[4] D. Hill and J. Wait, "Subsurface electromagnetic fields of a grounded cable of finite length," Canadian Journal of Physics, vol. 51, no. 14, pp. 1534-1540, 1973.

[5] J. Wait, "The electromagnetic fields of a horizontal dipole in the presence of a conducting half-space," Canadian Journal of Physics, vol. 39, no. 7, pp. 10171028, 1961.

[6] M. A. Persinger, ELF and VLF electromagnetic field effects, New York: Plenum Press, 1974.

[7] L. Yan, J. Waynert and C. Sunderman, "Measurements and modeling of through-the-earth communications for coal mines," IEEE Transaction on Industry Applications, vol. 49, no. 5, pp. 1979-1983, 2013.

[8] D. A. Hill, "Electromagnetic surface fields of an inclined buried cable of finite length," Journal of Applied Physics, vol. 44, no. 12, pp. 5275-5279, 1973.

[9] N. Damiano, B. Whisner, L. Yan and C. Zhou, "Simulation and measurement of electric field, low frequency signals through the earth using copper-clad, steel ground rods," in IEEE IAS, 2016. 